\title{
Role of Alkaline Serine Protease, Asp, in Vibrio alginolyticus Virulence and Regulation of Its Expression by LuxO-LuxR Regulatory System
}

\author{
Rui, Haopeng ${ }^{1}$, Qin Liu ${ }^{1}$, Qiyao Wang ${ }^{1}$, Yue Ma ${ }^{1}$, Huan Liu ${ }^{1}$, Cunbin Shi ${ }^{2}$, and Yuanxing Zhang ${ }^{*}$ \\ ${ }^{1}$ State Key Laboratory of Bioreactor Engineering, East China University of Science and Technology, Shanghai 200237, P. R. China \\ ${ }^{2}$ Pearl River Fisheries Research Institute, Chinese Academy of Fishery Science, Guangzhou 510380, P. R. China
}

Received: July 1, 2008 / Revised: September 23, 2008 / Accepted: September 26, 2008

The alkaline serine protease asp, which was shown to be a virulence factor of Vibrio alginolyticus as a purified protein, was cloned from $V$. alginolyticus EPGS, a strain recently isolated from moribund Epinephelus coioides in an outbreak of vibriosis in a mariculture farm of Shenzhen. The asp null mutant was constructed by homologous recombination with suicide plasmid pNQ705-1. Compared with the wild-type strain, the asp null mutant exhibited a significant decrease of total extracellular protease activity, and caused a 15-fold decrease in virulence of $V$. alginolyticus. In our previous study, the $\operatorname{lux} O$ and $l u x R_{\text {val }}$ genes from $V$. alginolyticus MVP01 were cloned and identified, and the luxO-lux $R_{v a l}$ regulatory couple was shown to regulate various genes expression, suggesting that it played a central role in the quorum sensing system of $V$. alginolyticus. In this study, the regulation of the asp gene was analyzed by using RT-PCR and quantitative real-time PCR methods; we proved that its transcription was greatly induced at the late stage of growth and was regulated by a luxO-luxR $R_{\text {val }}$ regulatory system.

Keywords: Alkaline serine protease, asp, quorum sensing, Vibrio alginolyticus

Quorum sensing, a type of cell-cell signaling, has been studied widely in recent years [3]. A typical quorum sensing system of Gram-negative bacteria has two types of autoinducers: acylated homoserine lactones (AI-1) and furanosyl borate diester (AI-2) [4, 28]. In Vibrio harveyi, detection of and response to AI-1 and AI-2 occurs through two parallel two-component signal transduction circuits, and a shared response regulator called LuxO integrates the information from these two circuits and relays it to LuxR, whereupon activated LuxR regulates the expression of various genes [20]. Vibrio spp. could use this system to

*Corresponding author

Phone: +86-21-64253065; Fax: +86-21-64253025;

E-mail: yxzhang@ecust.edu.cn control many processes including bioluminescence, virulence, mobility, biofilm formation, and extracellular polysaccharide production [32]. Significantly, extracellular proteases, the virulence factors of Vibrio spp., were shown to be regulated by their respective quorum sensing systems, such as hemagglutinin protease hap in V. cholerae [13], metalloprotease empA in $V$. anguillarum [6], and metalloprotease $v v p$ and cytolysin $v v h A$ in $V$. vulnificus [25].

Vibrio alginolyticus, a ubiquitous organism in seawater, has been isolated from different organisms as part of the normal marine flora. However, it has also been suggested that this species was a pathogen of several marine animals and humans. In the South China Sea, V. alginolyticus was reported to be the dominant causative agent of highmortality vibriosis in the large yellow croaker, sea bream, grouper, kuruma prawn, as well as shellfish species [16, 17].

Compared with other Vibrio spp., such as $V$. cholerae, $V$. anguillarum, and $V$. vulnificus, much less is known about the pathogenic mechanism of $V$. alginolyticus. Studies on the pathogenicity of various isolates of $V$. alginolyticus suggested that some extracellular enzymes with strong proteolytic activities could be important virulent factors $[1,2,11]$, and alkaline serine protease from the extracellular products (ECP) of V. alginolyticus was reported to be lethal to fish but the detailed information about its DNA sequence and protein structure was unknown [14]. By searching the whole genome of $V$. alginolyticus $12 \mathrm{G} 01$, we found that two alkaline serine proteases existed in the genome, and they were totally different from each other (16\% identity at the amino acid level). Blast analysis showed that one of the two alkaline serine proteases in the genome of $V$. alginolyticus $12 \mathrm{G} 01$ was homologous to ProA, which was only expressed in medium containing calcium and shown to be regulated by the quorum sensing system in $V$. alginolyticus MVP01 [23], and the other one was homologous to Asp, which was recently shown to be lethal to fish as purified protein with a $\mathrm{LD}_{50}$ of $0.25 \mu \mathrm{g}$ protein per gram of body weight and thought to be important in the virulence mechanism of $V$. alginolyticus [5]. 
In previous work, we demonstrated that $V$. alginolyticus MVP01 possessed the quorum sensing system involved in regulating extracellular proteases $[23,31]$. In this study, a new $V$. alginolyticus strain termed EPGS was isolated from moribund Epinephelus coioides in a recent outbreak of vibriosis in a mariculture farm of Shenzhen, a southern coastal city of China. We found that the extracellular protease activity of this strain was much stronger than that of the previous $V$. alginolyticus strain MVP01, but to our surprise, it only contained the protease gene asp and lost gene proA (data not shown). Thus, a null mutant of gene asp was constructed by allelic exchange, and the fish infection experiments and cytotoxicity assays showed that Asp plays a role in the pathogenic mechanism of $V$. alginolyticus EPGS. Furthermore, the expression of gene asp was proven to be regulated by the quorum sensing system.

\section{Materials ANd Methods}

\section{Bacterial Strains, Plasmids, and Growth Conditions}

Bacterial strains and plasmids are listed in Table 1. V. alginolyticus strains were grown at $30^{\circ} \mathrm{C}$ in Luria-Bertani medium supplemented with $3 \% \mathrm{NaCl}$ (LBS). Escherichia coli strains were grown at $37^{\circ} \mathrm{C}$ in Luria-Bertani medium with $1 \% \mathrm{NaCl}$. Antibiotics, when needed, were added to the media at the following final concentrations: $100 \mu \mathrm{g} / \mathrm{ml}$ ampicillin (Amp) and $34 \mu \mathrm{g} / \mathrm{ml}$ chloramphenicol (Cm) for E. coli; $100 \mu \mathrm{g} / \mathrm{ml} \mathrm{Amp} \mathrm{and} 7 \mu \mathrm{g} / \mathrm{ml} \mathrm{Cm}$ for V. alginolyticus.

\section{DNA Manipulation}

All molecular techniques were performed as previously described [24]. Enzymatic reactions and plasmid purifications were performed according to the manufacturer's instructions (TaKaRa, Dalian,
China). DNA sequencing and primer synthesis were carried out by Invitrogen Co. Ltd. (Shanghai, China).

\section{Construction of an Alkaline Serine Protease asp Mutant}

Primers used for the mutant construction are given in Table 2. The insertion mutant of gene asp was constructed as previously described [31]. Briefly, an internal fragment of the gene asp (600 bp) was amplified by PCR from $V$. alginolyticus EPGS chromosome with primers AspmutF and AspmutR. The PCR product was treated with SacI/SpeI restriction enzymes and cloned into the corresponding restriction sites of suicide plasmid pNQ705-1 [6]. The resulting plasmid pNQ-asp was transferred from E. coli to $V$. alginolyticus by bacterial mating, which used $E$. coli SM10 $\lambda$ pir as the donor strain [15], and the mating was allowed to progress on an LB agar plate at $30^{\circ} \mathrm{C}$ for $12 \mathrm{~h}$. The exconjugants with the plasmid integrated into the chromosome by homologous recombination were selected on LBS agar medium containing Cm $(7 \mu \mathrm{g} / \mathrm{ml})$ and Amp $(100 \mu \mathrm{g} / \mathrm{ml})$. The resulting mutant (NESP) was confirmed by PCR using primer pairs $\mathrm{AmF} / \mathrm{pNQF}$ and $\mathrm{AmR} / \mathrm{pNQR}$, and subsequent DNA sequencing.

The quorum sensing targeted genes $\operatorname{lux} O_{\text {val }}$ and $\operatorname{lux} R_{\text {val }}$ of $V$. alginolyticus EPGS were amplified by PCR, and the DNA sequencing results showed that they shared $100 \%$ identity to those of $V$. alginolyticus MVP01. Thus, we used the previous suicide plasmids pDM-luxO and pDM-luxR $[23,31]$ to construct in-frame deletion mutants in $l u x O_{v a l}$ and $l u x R_{v a l}$, respectively. The plasmids were mated by conjugation from $E$. coli SM10 $\lambda$ pir to wild-type $V$. alginolyticus EPGS. After allelic exchange, the absence of targeted fragments in the mutants was confirmed by PCR amplification using primers pairs LuxO-F/LuxO-R and LuxR-F/LuxR-R, and subsequent DNA sequencing. For genetic complementation of the $\operatorname{lux}_{\text {val }}$ mutant, the plasmid pMMB-luxR [31] with intact $\operatorname{lux} R_{\text {val }}$ gene and promoter region was introduced into the $\operatorname{lux} R_{v a l}$ mutant by conjugation. The complemented strain with the presence of the plasmid was selected and confirmed by PCR analysis.

Table 1. Strains and plasmids used in this study.

\begin{tabular}{|c|c|c|}
\hline Strain or plasmid & Characteristics & Reference or source \\
\hline \multicolumn{3}{|l|}{ Vibrio alginolyticus } \\
\hline EPGS & Amp; Pathogenic isolate from the aquiculture farm of the South China Sea & Lab collection \\
\hline NESP & Amp; EPGS, null mutant in asp & This study \\
\hline DEO & Amp; EPGS, in-frame deletion in $\operatorname{lux} O_{v a l}$ & This study \\
\hline DER & Amp; EPGS, in-frame deletion in lux $R_{v a l}$ & This study \\
\hline CDER & Amp; EPGS, complementation in trans with intact $l u x R_{v a l}$ gene & This study \\
\hline \multicolumn{3}{|l|}{ Escherichia coli } \\
\hline CC118 גpir & $\begin{array}{l}\text { Apir lysogen of CC118 ( } \Delta \text { (ara-leu) araD } \Delta \text { lacX74 galE galK phoA20 thi-1 rpsE } \\
\quad \text { rpoB argE(Am) recAl) }\end{array}$ & Dennis and Zylstra [10] \\
\hline SM10 $\lambda$ pir & $\mathrm{Km}^{\mathrm{r}}$; thi thr leu tonA lacY supE recA RP4-2-Tc::Mu $\lambda::$ pir & Liang et al. [15] \\
\hline Top 10 & $\mathrm{~F}^{\prime}[$ lacIq,Tn10(TetR)] mcrA $\Phi 80$ lacZ $\Delta \mathrm{m} 15 \Delta l a c$ X74 deoR recA1 & Invitrogen \\
\hline \multicolumn{3}{|c|}{ [ } \\
\hline pNQ705-1 & $\mathrm{Cm}^{\mathrm{r}}$; suicide vector that contains an R6K origin (pir requiring) & Croxatto et al. [6] \\
\hline pNQ-asp & $\mathrm{Cm}^{\mathrm{r}}$; pNQ705-1 derivative containing a 601 bp internal fragment of gene $a s p$ & This study \\
\hline pMD-luxO & $\begin{array}{l}\mathrm{Cm}^{\mathrm{r}} ; \mathrm{pDM} 4 \text { derivative containing } \operatorname{lu} x O \text { bp } 1-123 \text { fused in-frame to bp } 1281- \\
1362\end{array}$ & Wang et al. [31] \\
\hline pDM-luxR & $\mathrm{Cm}^{\mathrm{r}} ; \mathrm{pDM} 4$ derivative containing $l u x R_{v a l}$ bp 0-56 fused in-frame to bp 474-615 & Rui et al. [23] \\
\hline pMMB-luxR & $\mathrm{Cm}^{\mathrm{r}} ; \mathrm{pMMB} 206$ derivative containing lux $R_{v a l}$ bp from -782 to 1408 & Rui et al. [23] \\
\hline
\end{tabular}


Table 2. Primers used for cloning and qRT-PCR.

\begin{tabular}{ll}
\hline Primer & \multicolumn{1}{c}{ Sequence (5'-3') } \\
\hline AspmutF & TCAGAGCTCCGAACTGAGCAAAAT \\
AspmutR & ACGACTAGTCGTTACCGAAACCATA \\
AmF & CTAACGTTGTGAACATGAGCC \\
AmR & GACCAACATACCCCAATAGCT \\
LuxO-F & CTACGAGCGCAGTTCATCGCA \\
LuxO-R & GTCTATGGACATCGCCAGTTCACAA \\
LuxR-F & CACGCAAACGATCACCTAA \\
LuxR-R & TATCCACGCTCACCCAACT \\
pNQF & GGTGCTCCAGTGGCTTCTGTTTCTA \\
pNQR & CAGCAACTTAAATAGCCTCTAAGGT \\
AspM-F & GGTAACAACAATGCCCACGG \\
AspM-R & GCCCAGATGAGTAACCCCAAC \\
$16 S-F$ & AAAGCACTTTCAGTCGTGAGGAA \\
$16 S-R$ & TGCGCTTTACGCCCAGTAAT \\
\hline
\end{tabular}

\section{Measurement of Total Extracellular Protease Activity}

Protease activity of culture supernatant was determined by two methods. For qualitative assay, $V$. alginolyticus strains were grown for $12 \mathrm{~h}$ on LBS agar plates containing 1\% skimmed milk (SMLB; Oxoid, U.K.) and the clearing zones around the colonies were observed [29]. For quantitative assay, the strains were grown in LBS medium at $30^{\circ} \mathrm{C}$ for $9 \mathrm{~h}$, and protease activity was determined by the method described elsewhere $[9,14]$. Briefly, the bacterial cultures were harvested and filtered through $0.22-\mu \mathrm{m}$ filters (Millipore, U.S.A.), and the filtered supernatants $(0.5 \mathrm{ml})$ were incubated with the substrate hide powder azure (HPA; Sigma-Aldrich, U.S.A.) in $1.5 \mathrm{ml}$ of PBS buffer (pH 7.2) at $37^{\circ} \mathrm{C}$ for $2 \mathrm{~h}$. After stopping the reaction by trichlorocetic acid (TCA), the absorbance of the supernatant was assayed at $600 \mathrm{~nm}$. When needed, the serine protease inhibitor phenylmethylsulfonyl fluoride (PMSF) was added to reach a final concentration of $1 \mathrm{mmol} / \mathrm{l}$.

Gelatin-gel zymographic analysis was also used to characterize extracellular protease activity. V. alginolyticus strains were cultured in LBS medium for $9 \mathrm{~h}$ and the culture supernatants were harvested and filtered through $0.22-\mu \mathrm{m}$ filters (Millipore, U.S.A.). Two $\mu$ of each cell-free supernatant was mixed with nonreducing buffer and loaded in a $12 \%$ denaturing polyacrylamide gel copolymerized with $0.1 \%$ gelatin. After electrophoresis, gels were washed in $2.5 \%$ Triton X-100 for $1 \mathrm{~h}$ and incubated in $50 \mathrm{mmol} / 1$ Tris- $\mathrm{HCl}(\mathrm{pH} 8.0)$ buffer containing $5 \mathrm{mmol} / / \mathrm{CaCl}_{2}$ and $1 \mu \mathrm{mol} / / \mathrm{ZnCl}_{2}$ for $3 \mathrm{~h}$. Protein bands with gelatinase activity in the gels were visualized by Coomassie blue staining.

\section{Fish Infection}

Zebra fish (Brachydanio rerio) with an approximate weight of $1 \mathrm{~g}$ were infected with $V$. alginolyticus strains via route of intramuscular injection (i.m.) as described previously [33]. Four different bacterial dilutions varying by 10 -fold were used and seven fish were infected for each dilution. Death due to vibriosis was determined by the observation of gross clinical signs and confirmed by isolation of $V$. alginolyticus strains from infected organs of dead fish. The mortality of the fish was recorded over a period of 2 weeks. The $\mathrm{LD}_{50}$ values were calculated by a method described before [22].

\section{Cytotoxicity Assay}

Cytotoxicity of $V$. alginolyticus strains was assayed with epithelioma papillosum cyprini (EPC) cells as described previously [2,21]. The
EPC cells were grown as a monolayer at $25^{\circ} \mathrm{C}$ in Eagle's minimum essential medium (MEM; Sigma) supplemented with $10 \%$ fetal calf serum, and harvested with trypsin ethylenediaminetetraacetic acid. A $900-\mu 1$ aliquot of the cell suspension was inoculated to each well in a 24-well culture plate (Costar, U.S.A.). After incubation for $24 \mathrm{~h}$, $100 \mu \mathrm{l}$ of filtered supernatant of $V$. alginolyticus culture was added to EPC cell culture, and the EPC cells were inspected under microscopy for the morphologic damage. Cytotoxicity was determined by the MTT [3-(4,5-dimethylthiazol-2-yl)-2,5-diphenyltetrazolium bromide] method (Amersco, U.S.A.) after 24-h infection. Cell culture of the control group was treated with fresh LBS medium. Percent viability was calculated as follows: (optical density at $570 \mathrm{~nm}\left[\mathrm{OD}_{570}\right]$ of control group- $\mathrm{OD}_{570}$ of treated group) $/ \mathrm{OD}_{570}$ of control group $\times 100 \%$.

\section{RNA Isolation and Quantitative Real-Time RT-PCR}

Primers for quantitative real-time PCR are listed in Table 2. $V$. alginolyticus strains were grown in LBS medium at $30^{\circ} \mathrm{C}$, and RNA was isolated using an RNA isolating kit (Tiangen) and then treated with RNase-free DNaseI (Promega, U.S.A.) to exclude genomic DNA contamination. Equal amounts of RNA $(1 \mu \mathrm{g})$ were used to generate cDNA (BioToKo) with a random primer (TaKaRa). The synthesized cDNA was used as a template for PCR amplification. The primers AspM-F and AspM-R were used to amplify the internal segment of gene asp. 16S rRNA was selected as a control gene and amplified with primers $16 \mathrm{~S}-\mathrm{F}$ and $16 \mathrm{~S}-\mathrm{R}$. The quantitative real-time RT-PCR (qRT-PCR) reaction system contained $0.5 \mu \mathrm{l}$ of cDNA template, $1 \mu \mathrm{l}$ of the gene-specific primers mentioned above, $12.5 \mu \mathrm{l}$ of SYBR Green master mix (BioToKo), and $\mathrm{ddH}_{2} \mathrm{O}$ up to $25 \mu \mathrm{l}$ of total volume. qRT-PCR assays were performed in triplicate with a FTC-2000 detector (Funglyn Biotech, China) and each qRT-PCR experiment was repeated three times. Transcriptional levels were normalized to $16 \mathrm{~S}$ rRNA in each sample by the $\Delta \Delta \mathrm{C}_{\mathrm{T}}$ method [18].

\section{Nucleotide Sequence Accession Number}

The nucleotide sequence of alkaline serine protease gene asp from $V$. alginolyticus EPGS has been deposited in the GenBank under Accession No. EU484572.

\section{Results}

\section{Construction of a Null Mutation Within Gene asp}

The asp gene of $V$. alginolyticus EPGS was amplified by PCR and sequenced. The ORF contains 2,031 nucleotides and the deduced protein sequence shows $98 \%, 92 \%, 76 \%$, and $72 \%$ homology to those of $V$. alginolyticus $12 \mathrm{G} 01$, V. parahaemolyticus 2210633, V. alginolyticus HY9901, and $V$. harveyi HY01. The asp deficient mutant (NESP) was generated by integrating into the chromosome a mobilizable suicide plasmid, pNQ-asp, which contained a 600-bp PCR fragment from the encoding region of the gene asp. Primer pairs $\mathrm{AmF} / \mathrm{pNQF}$ and $\mathrm{AmR} / \mathrm{pNQR}$, located on chromosome and plasmid pNQ705-1, respectively, were chosen for PCR. Two PCR fragments were generated from the asp null mutant, whereas the wild-type EPGS yielded no PCR product (Fig. 1). These two PCR fragments were sequenced, and the result showed the correct integration. 
The quorum sensing targeted genes $\operatorname{lux} O_{v a l}$ and $\operatorname{lux} R_{v a l}$ of $V$. alginolyticus EPGS were cloned and the DNA sequencing result showed that they shared $100 \%$ identity to those of $V$. alginolyticus MVP01. Thus, the recombinant plasmids pDM-luxO and pDM-luxR were used respectively for constructing in-frame deletion mutant strains DEO $\left(\Delta l u x O_{v a l}\right)$ and DER $\left(\Delta l u x R_{v a l}\right)$. For genetic complementation of strain DER, plasmid pMMB-luxR was introduced by conjunction and the transconjugant $\left(l u x R_{v a l}{ }^{+}\right)$was designated as strain CDER (data not shown).

\section{Extracellular Protease Activity of Wild-Type EPGS and Null Mutant NESP}

The extracellular protease activity of mutant strain NESP was examined and shown to be much less than that of wildtype strain EPGS. As seen in Fig. 2A, the clearing zone of strain NESP was smaller than that of strain EPGS. To confirm this result, the total extracellular protease activities of 9-h cultures were determined with HPA as substrate. Compared with strain NESP, the strain EPGS exhibited much higher protease activity. However, when specific serine protease inhibitor PMSF was added to the supernatants of both strains, the protease activity of strain EPGS was dramatically decreased while the protease activity of strain NESP had a little change (Fig. 2B). The similar growth profiles of both strains in LBS medium (data not shown) suggested that impairment of extracellular protease production was not caused by growth defect. Zymography revealed that wild-type $V$. alginolyticus had strong proteolytic bands at

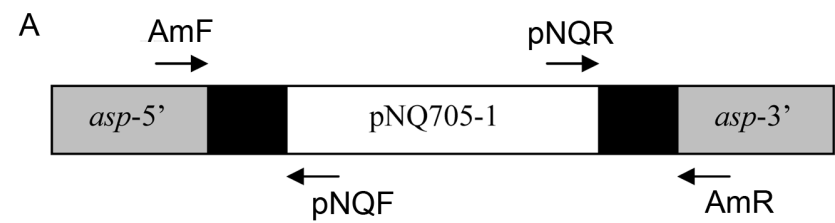

B

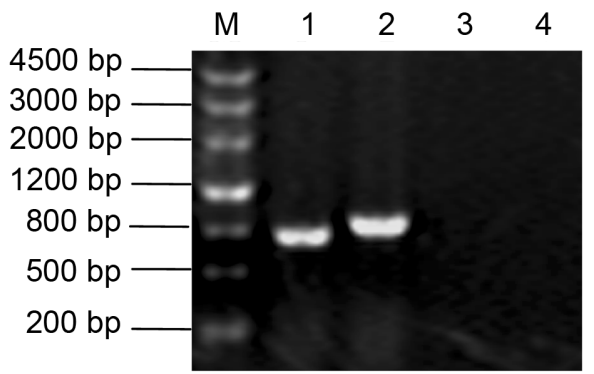

Fig. 1. Identification of the asp mutation.

A. A diagram of the chromosomal integration of plasmid pNQ-asp into the asp gene. The gray blocks represent the asp gene, and the black blocks represent the cloned 600-bp PCR fragment from the internal region of the asp gene that was used for homologous recombination. B. PCR screening and confirming of the asp null mutant. Primer pairs $\mathrm{AmF} / \mathrm{pNQF}$ and $\mathrm{AmF} /$ pNQR were utilized to amplify the DNA regions. Lanes 1,2: chromosomal DNA of the mutant NESP; lanes 3,4: chromosomal DNA of wild-type strain; lane M: DNA molecular weight marker. about $40 \mathrm{kDa}$, which clearly disappeared in the asp mutant. It also suggested that Asp was a dominant protease in the culture supernatant of $V$. alginolyticus and had more than one gelatin-degrading band (Fig. 2C). This was not unexpected,

A

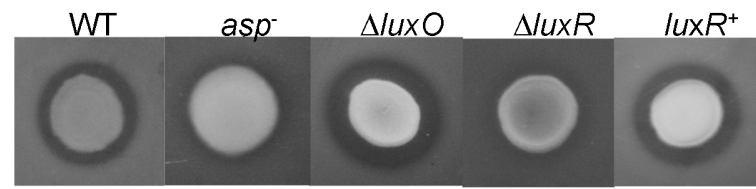

B

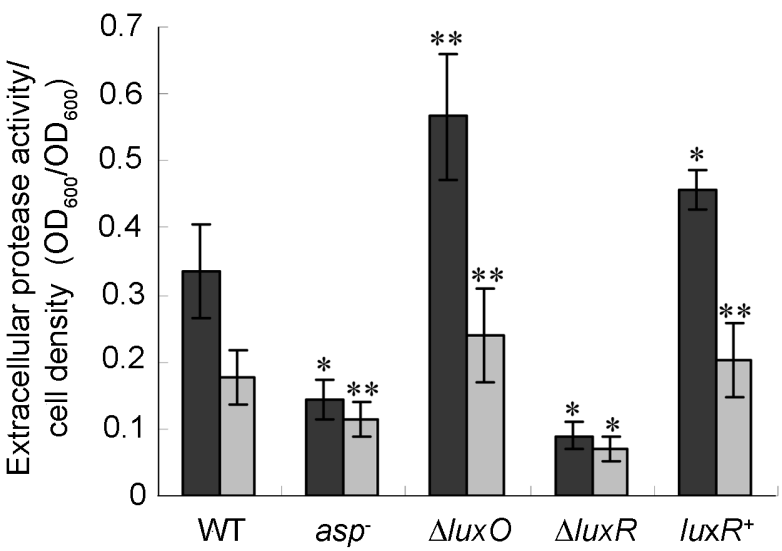

C $\mathrm{kDa} \quad \mathrm{WT}$ asp $\Delta / \mathrm{uxO} \quad \Delta / \mathrm{uxR} \quad \mathrm{lux} R^{+}$

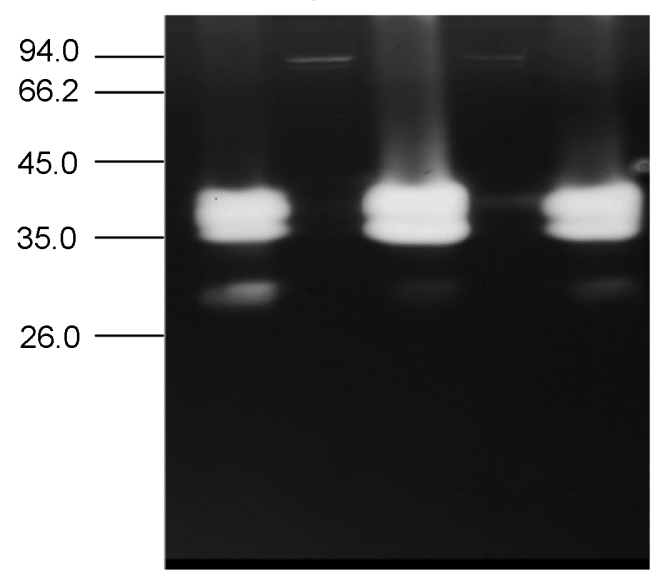

Fig. 2. Detection of extracellular protease activity.

A. Qualitative assay of extracellular protease activity on SMLB agar plates. V. alginolyticus strains of wild-type EPGS (WT), NESP (asp ${ }^{-}$), DEO $\left(\Delta l u x O_{v a l}\right)$, DER $\left(\Delta l u x R_{v a l}\right)$, and CDER $\left(l u x R_{v a l}{ }^{+}\right)$were grown on SMLB agar plates for $12 \mathrm{~h}$. Clearing zones around the colonies were observed. B. Extracellular protease activity measured by HPA digestion as described in Materials and Methods. V. alginolyticus strains were cultured in LBS medium for $9 \mathrm{~h}$. To each sample, a solution of PMSF (gray columns) or an equal volume of $\mathrm{ddH}_{2} \mathrm{O}$ (black columns) was added. The values shown are the means of three independent experiments, and the error bars represent the standard deviations. Data marked with one asterisk indicate $p$ value $<0.01$, and data with two asterisks indicate $p$-value $<0.05$ (Student $t$ test). C. Proteolytic activity was detected using a $12 \%$ SDS-PAGE gel copolymerized with $0.1 \%$ gelatin. Lane $1: V$. alginolyticus wild-type; lane 2 : asp mutant; lane 3: $\Delta l u x O_{v a l}$ mutant; lane 4: $\Delta l u x R_{v a l}$ mutant; lane 5: $\Delta l u x R_{v a l}$ mutant carrying plasmid pMMB-luxR. 
Table 3. Effect of the asp mutation on the $\mathrm{LD}_{50}$ and cytotoxicity of $V$. alginolyticus.

\begin{tabular}{cccccc}
\hline Strain & $\begin{array}{c}\text { Dose/fish } \\
(\text { CFU })\end{array}$ & $\begin{array}{c}\text { Mortality } \\
(\text { no. dead/no. injected })\end{array}$ & $\begin{array}{c}\text { Day of death } \\
\text { (no. dead/no. injected) }\end{array}$ & $\begin{array}{c}\text { LD }_{50} \text { value } \\
(\text { CFU/ml) }\end{array}$ & $\begin{array}{c}\text { Cytotoxicity } \\
(\%)\end{array}$ \\
\hline EPGS & $1 \times 10^{7}$ & $7 / 7$ & $1(3 / 7) 2(6 / 7) 4(7 / 7)$ & $6 \times 10^{5}$ & 57 \\
& $1 \times 10^{6}$ & $6 / 7$ & $1(1 / 7) 2(4 / 7) 4(6 / 7)$ & & \\
& $1 \times 10^{5}$ & $4 / 7$ & $2(1 / 7) 3(2 / 7) 4(3 / 7) 6(4 / 7)$ & & \\
NESP & $1 \times 10^{4}$ & $1 / 7$ & $4(1 / 7)$ & $1 \times 10^{7}$ & 32 \\
& $1 \times 10^{7}$ & $6 / 7$ & $1(2 / 7) 2(6 / 7)$ & & \\
& $1 \times 10^{6}$ & $3 / 7$ & $2(3 / 7)$ & & \\
Control & $1 \times 10^{5}$ & $0 / 7$ & NA* & & \\
\hline
\end{tabular}

*NA, not applicable: no death due to vibriosis during the 14-day experiment.

because there was evidence that the extracellular alkaline serine proteases might be degraded as they were secreted from the cytoplasm $[1,12,14,19]$.

\section{Role of Asp as a Virulence Factor}

In order to investigate the role of alkaline serine protease in the $V$. alginolyticus virulence mechanism, zebra fish (Brachydanio rerio) was used as the fish model to assay the virulence difference between the wild-type EPGS and the asp null mutant. As showed in Table 3, the intramuscular $\mathrm{LD}_{50}$ values were $1.0 \times 10^{7}$ bacteria for asp null mutant and $6 \times 10^{5}$ bacteria for wild-type EPGS, respectively. The asp mutation led to a 15 -fold decrease in strain virulence, indicating that Asp has a role in the pathogenic mechanism of $V$. alginolyticus.

The cytotoxicity of $V$. alginolyticus strains against EPC cells was further assessed by the MTT method. After 24-h infection, the cytotoxic effects of extracellular products produced by $V$. alginolyticus strains were analyzed. As shown in Table 3, compared with the parent strain, the mutant strain NESP exhibited a $45 \%$ decrease of cytotoxicity against EPC cells. The morphological changes of cell monolayer could be observed after $8 \mathrm{~h}$ of infection in both strains, and the changes mainly involved cell rounding, shrinking, detachment, and finally monolayer destruction.

\section{Growth-Dependent Expression of asp in $V$. alginolyticus EPGS}

Since the maximum extracellular protease activity of wild-type EPGS was observed at the late stage of growth (data not shown), it was of interest to investigate whether the expression of alkaline serine protease in $V$. alginolyticus EPGS was growth-dependent. RT-PCR and quantitative real-time RT-PCR were used to measure the asp mRNA transcripts at different growth stages. Figs. 3A and 3B showed that asp transcriptional levels changed apparently during the whole growth period. A few asp mRNA transcripts were produced at the early stage of growth, whereas the amount of transcripts increased significantly (35- fold) at the late stage of growth. Obviously, the transcription of asp in V. alginolyticus was induced by cell density.

\section{Positive Regulation of asp Expression by Quorum Sensing}

The extracellular protease activities of the $V$. alginolyticus wild-type strain and quorum sensing mutants were examined. We found that the mutant $\Delta l u x R_{v a l}$ exhibited a $70 \%$ decrease whereas the mutant $\Delta l u x O_{v a l}$ exhibited a 2-fold increase of extracellular protease activity (Figs. 2A and 2B). This suggested that the total extracellular protease production was controlled by the quorum sensing system, which was consistent with the result previously described [23, 31].

Since the expression of asp in $V$. alginolyticus EPGS was induced at the late stage of growth, it was of concern whether expression of asp was regulated by the quorum sensing system. Zymography showed that the $\Delta l u x O_{v a l}$ mutant had strong proteolytic bands at about $40 \mathrm{kDa}$, which clearly disappeared in the $\Delta l u x R_{\text {val }}$ mutant and reappeared in $l u x R^{+}$(Fig. 2C). RT-PCR and quantitative real-time RT-PCR were then used to further examine $a s p$ transcription at the late stage of growth in different strains. As shown in Figs. 4A and 4B, compared with wild-type $V$. alginolyticus, the deletion mutant $\Delta l u x R_{\text {val }}$ exhibited a $92 \%$ decrease of asp transcripts, whereas the deletion mutant $\Delta l u x O_{v a l}$ exhibited an elevated level (3.3-fold) of asp transcripts, and the complemented strain $l u x R_{\text {val }}{ }^{+}$restored the expression of asp to the level of wild-type strain. Meanwhile, the transcriptional levels of control gene 16S rRNA were almost the same in all strains.

\section{Discussion}

Although two alkaline serine proteases have been found in the genome of $V$. alginolyticus $12 \mathrm{G} 01$, only the protease gene $a s p$ could be cloned from $V$. alginolyticus EPGS. Furthermore, we found that the deduced protein sequence of asp showed close homology to those of $V$. alginolyticus strain $12 \mathrm{G} 01$ and $V$. 
A

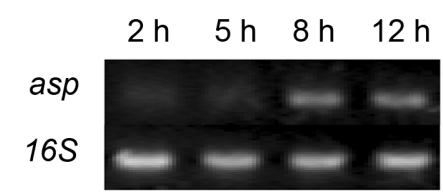

B

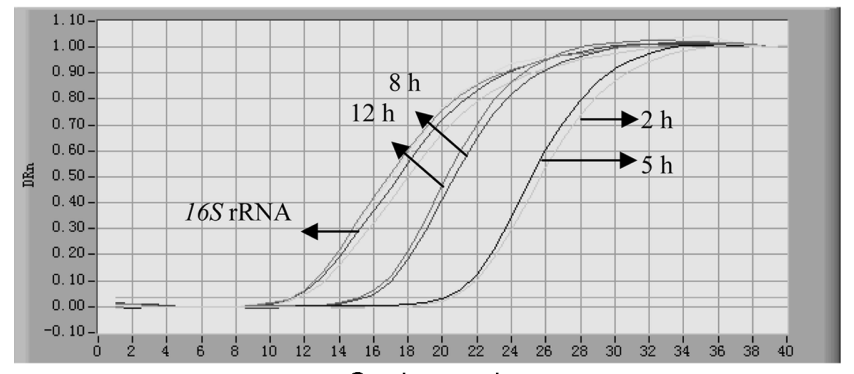

C

Cycle number

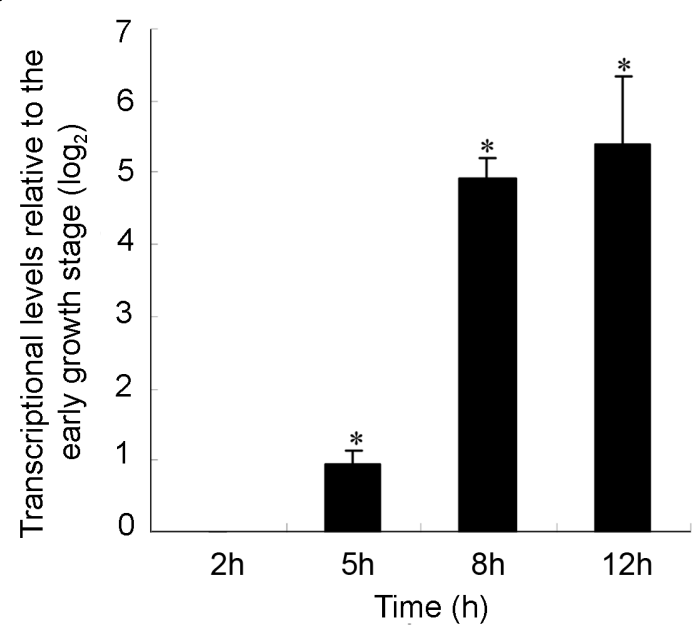

Fig. 3. The asp expression profile in the whole growth period. Overnight culture of wild-type $V$. alginolyticus EPGS was diluted into fresh LBS medium and then incubated at $30^{\circ} \mathrm{C}$. Transcription of $a s p$ at various growth stages was detected by RT-PCR (A) and quantitative real-time PCR (B). C. Transcriptional levels of asp at different growth stages are normalized to control gene 16S rRNA and are shown as $\log _{2}$ difference relative to the level of early growth stage. The values shown are an average of three independent experiments with the standard deviation, indicated by the error bars. Data marked with one asterisk represent that the asp expression is statistically different from that of early growth stage (Student $t$-test, $p<0.01$ ).

parahaemolyticus strain RIMD 2210633, but not to that of $V$. alginolyticus strain HY9901. These were not unexpected results because there was evidence that the extracellular alkaline serine proteases produced by $V$. alginolyticus might differ greatly in their sequences, depending on the strain, source of the isolate, and the medium $[1,8,12,19,30]$.

Phenylmethylsulfonyl fluoride (PMSF) is a covalently binding irreversible serine protease inhibitor. When added, the total extracellular protease activity of $V$. alginolyticus EPGS was largely inhibited, suggesting that the extracellular proteases of $V$. alginolyticus mainly consist of serine proteases. This was consistent with the result described previously [14].
A

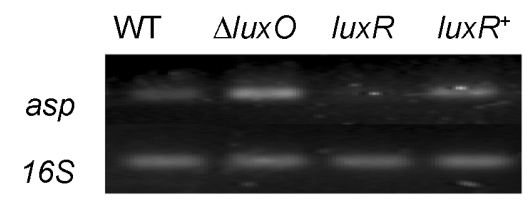

B

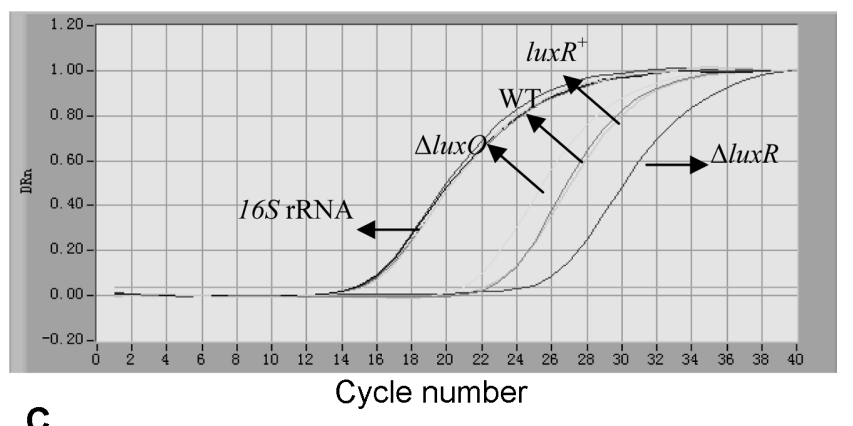

C

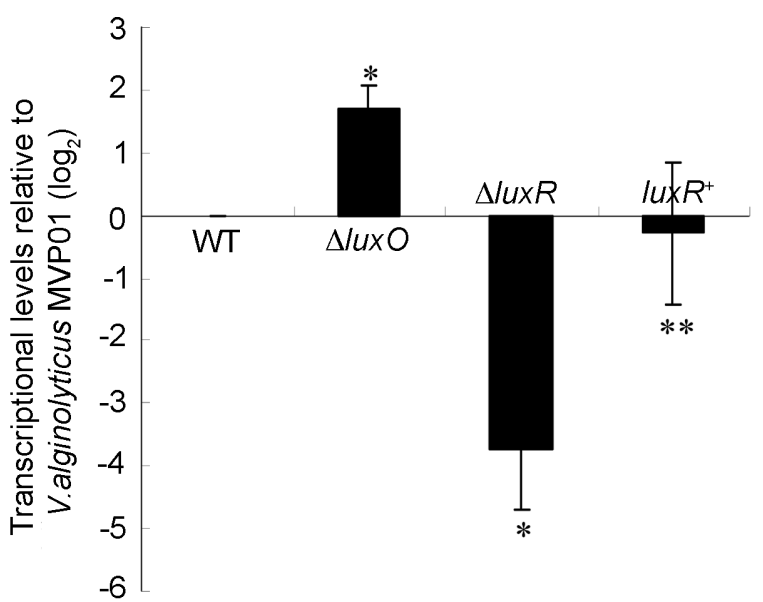

Fig. 4. Regulation of alkaline serine protease asp transcription by quorum sensing system.

A. V. alginolyticus strains EPGS (WT), DEO ( $\left.\Delta l u x O_{v a l}\right)$, DER ( $\left.\Delta l u x R_{v a l}\right)$, and CDER (lux $R_{\text {val }}{ }^{+}$) were cultured in LBS for $9 \mathrm{~h}$ and mRNA transcripts of asp were detected by RT-PCR. 16S rRNA was selected as a control. B. Transcriptional levels of asp at the stationary growth phase $(9 \mathrm{~h})$ were examined by quantitative real-time PCR. C. The transcriptional levels of asp in different mutant strains are normalized to control gene 16S rRNA and are shown as $\log _{2}$ difference relative to the levels of the wild-type. The values shown are an average of three independent experiments with the standard deviation, indicated by the error bars. Data marked with one asterisk represent that the asp expression is statistically different from that of wild-type cells (Student $t$-test, $p<0.01$ ), and data with two asterisks represent that the asp expression is not statistically different from that of wild-type cells (Student $t$-test, $p>0.05$ )

Based on our data, it was also found that, when gene asp was knocked out, the protease activity of $V$. alginolyticus was dramatically decreased, and inhibitor PMSF had a little effect on the protease activity of strain $a s p$. This indicates that the Asp is a major serine protease secreted by $V$. alginolyticus.

Although alkaline serine protease was believed to be a virulence factor of $V$. alginolyticus in some reports, a direct correlation of the loss of the alkaline serine protease to a decrease in virulence of $V$. alginolyticus was not established 
$[5,14]$. In our experiment of fish infection, wild-type $V$. alginolyticus strain EPGS killed about $60 \%$ zebra fish when administered by i.m. injection at a dose of $1 \times 10^{5}$ CFU per gram of fish body, whereas the asp null mutant strain NESP was completely avirulent to zebra fish at the same dose. Simultaneously, an obvious decrease in cytotoxicity to epithelioma papillosum cyprini (EPC) cells was also observed in the asp null mutant. The results confirm that the alkaline serine protease asp is important for the virulence of $V$. alginolyticus.

High transcription of the alkaline serine protease gene asp at the late stage of growth indicates that Asp may function only when the bacterial cells reach a high population density in fish. Proteases with such an expression pattern have been demonstrated to be regulated by the quorum sensing system in many pathogenic bacteria such as $P$. aeruginosa, $V$. cholerae, $V$. anguillarum, and $V$. mimicus [7, 26, 27, 34]. Existence of a $V$. harveyi-like quorum sensing system regulatory mechanism in $V$. alginolyticus has been proposed based on the detection of AI-2 activity in the culture supernatant and the identification of a $V$. harveyi-type LuxR homolog $[23,33]$. Here, we further demonstrated that asp transcription was regulated by a quorum sensing system, and the asp transcripts were greatly reduced in a LuxR $\mathrm{R}_{v a l}$ deficient mutant but increased in a $\mathrm{LuxO}_{v a l}$ deficient mutant. Furthermore, we also found that the disruption of $\operatorname{lux} O_{v a l}$ in $V$. alginolyticus resulted in a higher level of lux $R_{v a l}$ mRNA (data not shown). This indicates that $\mathrm{LuxO}_{v a l}$ negatively regulates the expression of luxR $R_{v a l}$, which in turn activates the expression of asp.

The regulation of extracellular protease by quorum sensing was found in some pathogenic bacteria, such as A. hydrophila and P. aeruginosa [26,29], in which the production of extracellular proteases was inhibited at low cell density and rapidly induced at high cell density. The similar regulation pattern of asp was also found in $V$. alginolyticus in this work. This type of regulation mechanism is very important and it can be used to escape host immunity at low cell density, since if extracellular protease is expressed too early, effective host defenses will be induced and the infection probably will be terminated.

In summary, our results demonstrated that the alkaline serine protease asp was a virulence factor in $V$. alginolyticus. Two pivotal regulators of quorum sensing systems in $V$. alginolyticus, $\mathrm{LuxO}_{v a l}$ and $\mathrm{LuxR}_{v a l}$, were involved in the regulation of alkaline serine protease $a s p$. Further investigation of the regulation mechanism of asp by the quorum sensing system in $V$. alginolyticus will be of interest.

\section{Acknowledgments}

We thank Prof. Debra Milton (Umeå University, Sweden) and Dr. Biao Kan (The Chinese Academy of Preventive
Medicine, China) for kindly sending strains used for the mutant construction in this study. This work was supported by the National Natural Science Foundation of China (No. U0633004).

\section{REFERENCES}

1. Aguirre-Guzman, G., H. Ruiz, and F. Ascencio. 2004. A review of extracellular virulence product of Vibrio species important in diseases of cultivated shrimp. Aquac. Res. 35: 1395-1404.

2. Balebona, M. C., M. J. Andreu, M. A. Bordas, I. Zorrilla, M. A. Morinigo, and J. J. Borrego. 1998. Pathogenicity of Vibrio alginolyticus for cultured gilt-head sea bream (Sparus aurata L.). Appl. Environ. Microbiol. 64: 4269-4275.

3. Bassler, B. L. and R. Losick. 2006. Bacterially speaking. Cell 125: 237-246.

4. Bassler, B. L., M. Wright, R. E. Showalter, and M. R Silverman. 1993. Intercellular signalling in Vibrio harveyi: Sequence and function of genes regulating expression of luminescence. Mol. Microbiol. 9: 773-786.

5. Cai, S. H., Z. H. Wu, J. C. Jian, and Y. S. Lu. 2007. Cloning and expression of the gene encoding an extracellular alkaline serine protease from Vibrio alginolyticus strain HY9901, the causative agent of vibriosis in Lutjanus erythopterus (Bloch). J. Fish Dis. 30: 493-500.

6. Croxatto, A., V. J. Chalker, J. Lauritz, J. Jass, A. Hardman, P. Williams, M. Camara, and D. L. Milton. 2002. VanT, a homologue of Vibrio harveyi LuxR, regulates serine, metalloprotease, pigment, and biofilm production in Vibrio anguillarum. J. Bacteriol. 184: 1617-1629.

7. Croxatto, A., J. Pride, A. Hardman, P. Williams, M. Camara, and D. L. Milton. 2004. A distinctive dual-channel quorumsensing system operates in Vibrio anguillarum. Mol. Microbiol. 52: 1677-1689.

8. Deane, S. M., F. T. Robb, S. M. Robb, and D. R. Woods. 1989. Nucleotide sequence of the Vibrio alginolyticus calcium-dependent, detergent-resistant alkaline serine exoprotease A. Gene 76: 281288 .

9. Denkin, S. M. and D. R. Nelson. 1999. Induction of protease activity in Vibrio anguillarum by gastrointestinal mucus. Appl. Environ. Microbiol. 65: 3555-3560.

10. Dennis, J. J. and G. J. Zylstra. 1998. Plasposons: Modular selfcloning minitransposon derivatives for rapid genetic analysis of Gram-negative bacterial genomes. Appl. Environ. Microbiol. 64: 2710-2715.

11. Gomez-Leon, J., L. Villamil, M. L. Lemos, B. Novoa, and A. Figueras. 2005. Isolation of Vibrio alginolyticus and Vibrio splendidus from aquacultured carpet shell clam (Ruditapes decussatus) larvae associated with mass mortalities. Appl. Environ. Microbiol. 71: 98-104.

12. Hare, P., T. Scott-Burden, and D. R. Woods. 1983. Characterization of extracellular alkaline proteases and collagenase induction in Vibrio alginolyticus. J. Gen. Microbiol. 129: 1141-1147.

13. Jobling, M. G. and R. K. Holmes. 1997. Characterization of hapR, a positive regulator of the Vibrio cholerae HA/protease gene hap, and its identification as a functional homologue of the Vibrio harveyi luxR gene. Mol. Microbiol. 26: 10231034. 
14. Lee, K. K., S. R. Yu, and P. C. Liu. 1997. Alkaline serine protease is an exotoxin of Vibrio alginolyticus in kuruma prawn, Penaeus japonicus. Curr. Microbiol. 34: 110-117.

15. Liang, W., S. Wang, F. Yu, L. Zhang, G. Qi, Y. Liu, S. Gao, and B. Kan. 2003. Construction and evaluation of a safe, live, oral Vibrio cholerae vaccine candidate, IEM108. Infect. Immun. 71: 5498-5504.

16. Liu, C. H., W. Cheng, J. P. Hsu, and J. C. Chen. 2004. Vibrio alginolyticus infection in the white shrimp Litopenaeus vannamei confirmed by polymerase chain reaction and $16 \mathrm{~S}$ rDNA sequencing. Dis. Aquat. Organ. 61: 169-174.

17. Liu, Q., P. Wang, Y. Ma, and Y. Zhang. 2007. Characterization of the Vibrio alginolyticus fur gene and localization of essential amino acid sites in Fur by site-directed mutagenesis. J. Mol. Microbiol. Biotechnol. 13: 15-21.

18. Livak, K. J. and T. D. Schmittgen. 2001. Analysis of relative gene expression data using real-time quantitative PCR and the $2_{\mathrm{T}}^{-\Delta \Lambda \mathrm{C}}$ method. Methods 25: 402-408.

19. Long, S., M. A. Mothibeli, F. T. Robb, and D. R. Woods. 1981. Regulation of extracellular alkaline protease activity by histidine in a collagenolytic Vibrio alginolyticus strain. J. Gen. Microbiol. 127: 193-199.

20. Miyamoto, C. M., P. V. Dunlap, E. G. Ruby, and E. A. Meighen. 2003. LuxO controls luxR expression in Vibrio harveyi: Evidence for a common regulatory mechanism in Vibrio. Mol. Microbiol. 48: 537-548.

21. Park, K. S., T. Ono, M. Rokuda, M. H. Jang, K. Okada, T. Iida, and T. Honda. 2004. Functional characterization of two type III secretion systems of Vibrio parahaemolyticus. Infect. Immun. 72: 6659-6665.

22. Reed, L. J. and H. Muench 1938. A simple method of estimating fifty percent end points. Am. J. Hyg. 27: 493-497.

23. Rui, H., Q. Liu, Y. Ma, Q. Wang, and Y. Zhang. 2008. Roles of LuxR in regulating extracellular alkaline serine protease A, extracellular polysaccharide and mobility of Vibrio alginolyticus. FEMS Microbiol. Lett. 285: 155-162.

24. Sambrook, J., E. Fritsch, and T. Maniatis. 1989. Molecular Cloning: A Laboratory Manual, 2nd Ed, Cold Spring Harbor Laboratory, Press Cold Spring Harbor, New York, U.S.A.
25. Shao, C. P. and L. I. Hor. 2001. Regulation of metalloprotease gene expression in Vibrio vulnificus by a Vibrio harveyi LuxR homologue. J. Bacteriol. 183: 1369-1375.

26. Storey, D. G., E. E. Ujack, H. R. Rabin, and I. Mitchell. 1998. Pseudomonas aeruginosa las $R$ transcription correlates with the transcription of las $A$, las $B$, and tox $A$ in chronic lung infections associated with cystic fibrosis. Infect. Immun. 66: 2521-2528.

27. Sultan, Z., S. Miyoshi, and S. Shinoda. 2006. Presence of LuxS/ AI-2 based quorum-sensing system in Vibrio mimicus: LuxO controls protease activity. Microbiol. Immunol. 50: 407-417.

28. Surette, M. G., M. B. Miller, and B. L. Bassler. 1999. Quorum sensing in Escherichia coli, Salmonella typhimurium, and Vibrio harveyi: A new family of genes responsible for autoinducer production. Proc. Natl. Acad. Sci. U.S.A. 96: 1639-1644.

29. Swift, S., M. J. Lynch, L. Fish, D. F. Kirke, J. M. Tomas, G. S. Stewart, and P. Williams. 1999. Quorum sensing-dependent regulation and blockade of exoprotease production in Aeromonas hydrophila. Infect. Immun. 67: 5192-5199.

30. Takeuchi, H., Y. Shibano, K. Morihara, J. Fukushima, S. Inami, B. Keil, A. M. Gilles, S. Kawamoto, and K. Okuda. 1992 Structural gene and complete amino acid sequence of Vibrio alginolyticus collagenase. Biochem. J. 281: 703-708.

31. Wang, Q., Q. Liu, Y. Ma, H. Rui, and Y. Zhang. 2007. LuxO controls extracellular protease, haemolytic activities and siderophore production in fish pathogen Vibrio alginolyticus. $J$. Appl. Microbiol. 103: 1525-1534.

32. Waters, C. M. and B. L. Bassler. 2005. Quorum sensing: Cellto-cell communication in bacteria. Annu. Rev. Cell Dev. Biol. 21: 319-346.

33. Ye, J., Y. Ma, Q. Liu, D. L. Zhao, Q. Y. Wang, and Y. X. Zhang. 2008. Regulation of Vibrio alginolyticus virulence by the LuxS quorum-sensing system. J. Fish Dis. 31: 161-169.

34. Zhu, J., M. B. Miller, R. E. Vance, M. Dziejman, B. L. Bassler, and J. J. Mekalanos. 2002. Quorum-sensing regulators control virulence gene expression in Vibrio cholerae. Proc. Natl. Acad. Sci. U.S.A. 99: 3129-3134. 